\title{
Medical treatment of octogenarians with chronic heart failure: data from CHECK-HF
}

\author{
Gerard C. M. Linssen ${ }^{1}$. Jesse F. Veenis ${ }^{2} \cdot$ Alexandra Kleberger $^{1} \cdot$ Marcel J. W. Grosfeld $^{3}$ - Eric P. Viergever ${ }^{4}$. \\ Bas M. van Dalen ${ }^{5}$. Wendy de Valk-Bedijn ${ }^{6}$. Jorina Langerveld ${ }^{7} \cdot$ Hans-Peter Brunner-La Rocca ${ }^{8} \cdot$ Arno W. Hoes $^{9}$. \\ Jasper J. Brugts ${ }^{2} \cdot$ for the CHECK-HF investigators
}

Received: 4 November 2019 / Accepted: 20 January 2020

(c) Springer-Verlag GmbH Germany, part of Springer Nature 2020

\begin{abstract}
Background Elderly heart failure (HF) patients are underrepresented in clinical trials, though are a large proportion of patients in real-world practice. We investigated practice-based, secondary care HF management in a large group of chronic HF patients aged $\geq 80$ years (octogenarians).

Methods We analyzed electronic health records of 3490 octogenarians with chronic HF at 34 Dutch outpatient clinics in the period between 2013 and 2016, 49\% women. Study patients were divided into HFpEF [LVEF $\geq 50 \% ; n=911(26.1 \%)$ ], HFrEF [LVEF < 40\%; $n=2009$ (57.6\%)] and HF with mid-range EF [HFmrEF: LVEF 40-49\%; $n=570(16.3 \%)$ ].

Results Most HFrEF patients aged $\geq 80$ years received a beta blocker and a renin-angiotensin system (RAS) inhibitor (angiotensin-converting enzyme (ACE) inhibitor or angiotensin receptor blocker), i.e. $78.3 \%$ and $72.8 \%$ respectively, and a mineralocorticoid receptor antagonist (MRA) was prescribed in $52.0 \%$ of patients. All three of these guideline-recommended medications (triple therapy) were given in only $29.9 \%$ of octogenarians with $\mathrm{HFrEF}$, and at least $50 \%$ of target doses of triple therapy, beta blockers, RAS inhibitor and MRA, were prescribed in $43.8 \%, 62.2 \%$ and $53.5 \%$ of the total group of HFrEF patients. Contraindications or intolerance for beta blockers was present in $3.5 \%$ of the patients, for RAS inhibitors and MRAs in, $7.2 \%$ and $6.1 \%$

Conclusions The majority of octogenarians with HFrEF received one or more guideline-recommended HF medications. However, triple therapy or target doses of the medications were prescribed in a minority. Comorbidities and reported contraindications and tolerances did not fully explain underuse of recommended HF therapies.
\end{abstract}

\section{Graphic abstract}

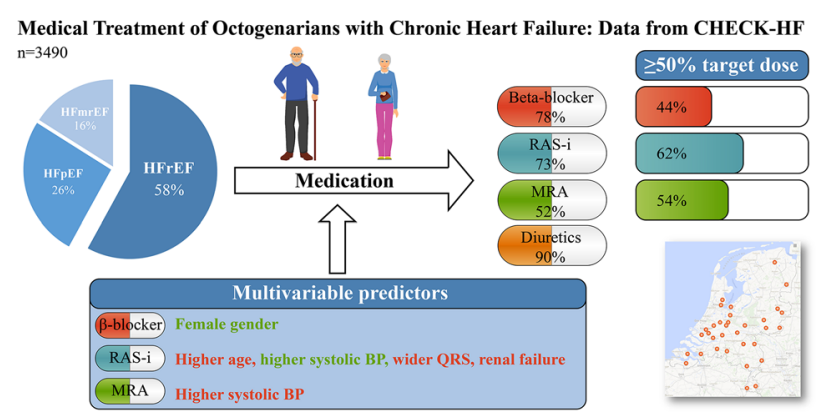

Jesse F. Veenis and Alexandra Kleberger contributed equally to this work.

Electronic supplementary material The online version of this article (https://doi.org/10.1007/s00392-020-01607-y) contains supplementary material, which is available to authorized users.

Extended author information available on the last page of the article 
Keywords Heart failure $\cdot$ Older people $\cdot$ Guidelines $\cdot$ Adherence $\cdot$ Medication

\section{Introduction}

Elderly patients represent a major proportion of the heart failure (HF) population. Most of these elderly patients have multiple morbidities [1-3]. This may complicate adherence to advocated HF management.

In general, optimizing guideline-recommended HF therapies improves quality of life, morbidity and mortality significantly [4-6]. However, randomized clinical trials investigating $\mathrm{HF}$ therapies did not represent the real-life HF population. The patients enrolled in these trials were on average 10 years younger than in real-world practice; elderly patients were largely underrepresented and very elderly were even excluded, except for the SENIORS-study [7-10].

As such, there are considerable gaps of knowledge in HF treatment effects in octogenarians. Practice guidelines do not provide age-specific recommendations for implementation and utilization of HF therapies [4, 5], but several registries reported lower prescription rates of evidence-based medication in the elderly [11-18]. High age-related factors, e.g., frailty, fall risk, cognitive impairment, dementia and disability, and also polypharmacy and concerns on drug interaction may interfere with initiation and persistence of HF medication, and as such are potential barriers for optimal therapy [5]. Importantly, detailed data regarding prescribed HF medication in the very elderly are scarce.

In a large-scale real-world registry at Dutch HF outpatient clinics, we investigated medical HF therapies and determinants of prescription of individual HF drugs in a substantial group of octogenarians $[19,20]$, better reflecting contemporary practice-based $\mathrm{HF}$ in secondary care.

\section{Methods}

The design and methods of the CHECK-HF (Chronisch Hartfalen ESC-richtlijn Cardiologische praktijk Kwaliteitsproject HartFalen) registry have been published in detail earlier [19]. Briefly, the CHECK-HF registry consists of 10,910 patients with chronic HF from a total of 34 participating Dutch centers. Between 2013 and 2016, all centers included patients diagnosed with HF based on the 2012 ESC Guidelines on HF (i.e., based on symptoms and echo parameters) who were seen at the outpatient HF clinic (96\%) or general cardiology outpatient clinic (4\%) if no specific HF clinic was present.

From electronic health records, baseline patient characteristics, etiology of HF, comorbidities, basic echocardiographic and electrocardiographic (ECG) parameters, laboratory markers, pacemaker, ICD and CRT treatment as well as prescription rates of medication (drug name, dosage and frequency and total daily dose) were recorded. The target doses of guideline-recommended HF medication are presented in Suppl. Table 1. Drug doses were calculated compared to the recommended dose and according to guidelines as a daily dose or percentage of actual recommended daily dose.

Furthermore, contraindications and intolerance as indicated by the treating physician were collected. No predefined rules were applied to determine absolute contraindications. CHECK-HF is a cross-sectional observational cohort study and there were no outcome data collected.

There were 3601 patients aged $\geq 80$ years, comprising $33.1 \%$ of the total CHECK-HF cohort.

In $111(3.1 \%)$ patients, recording of ejection fraction or age in the database was insufficient to classify patients; so, these patients were excluded from this analysis. In the current analyses of the remaining 3490 patients, aged $\geq 80$ years, we focused on the prescribed HF medication.

Based on echocardiographic results, octogenarians were divided based on LVEF or visual assessment of the function of the left ventricle (LV) according to the contemporary 2016 ESC HF Guidelines into HFpEF [LVEF $\geq 50 \% ; n=911$ (26.1\%)], HFrEF [LVEF < 40\%; $n=2009$ (57.6\%)] and HF with mid-range EF [HFmrEF: LVEF $40-49 \% ; n=570$ $(16.3 \%)]$.

This study was approved by the medical ethics committee 2017 at Maastricht University Medical Center (Maastricht, the Netherlands). No informed consent of the participants in this registry was required.

\section{Statistics}

Continuous data are expressed as mean value \pm SD or median and interquartile range, depending on the distribution of the data, and compared by applying one-way analysis of variances (ANOVA) or Mann-Whitney $U$-test. Categorical data are expressed as counts and percentages, and compared by the Pearson Chi-square test. A two-sided $p$ value of 0.05 was considered statistically significant.

Multivariable predictors for the use of HF medication were sought, using multivariable logistic regression analysis, using the stepwise backward procedure. All predictors of medication use in univariable analysis (data not shown) at a $p$ value of $<0.10$ were included in the multivariable regression analysis. Results of logistic regression are presented as odds ratio (ORs). Some missing data occurred in the variables included in the multivariable analyses, which we corrected using multiple imputation. If the missing 
variables showed a monotone pattern of missing values, the monotone method was used; otherwise, an iterative Markov chain Monte Carlo method was used with a number of 10 iterations. A total of 5 imputations were performed, and the pooled data were analyzed. The imputed data were only used for the multivariable analysis. For all reported data of the multivariable analysis, we compared crude and imputed $p$ values as well as the odds ratios and confidence intervals to analyze whether imputation changed the results, and if no significant changes occurred, we present the imputed values in the main analyses.
All analyses were performed with SPSS Statistical Package version 24.0 (SPSS Inc, Chicago, Illinois).

\section{Results}

Baseline characteristics of the $3490 \mathrm{HF}$ patients aged $\geq 80$ years are shown in Table 1 . The median [IQR] age was 84 [82.0-87.0] years and $49 \%$ were women. Most patients were in NYHA class II and approximately half of the patients had a ischemic cause of their HF. Median

Table 1 Characteristics of octogenarians with HF according to LVEF groups (ESC Guidelines 2016)

\begin{tabular}{|c|c|c|c|c|c|}
\hline & Total $(n=3490)$ & $\operatorname{HFrEF}(n=2009)$ & $\operatorname{HFmrEF}(n=570)$ & $\operatorname{HFpEF}(n=911)$ & $p$ value \\
\hline Age (years) $(n=3490)$ & $84.0[82.0-87.0]$ & $84.0[82.0-87.0]$ & $84.0[82.0-87.0]$ & $85.0[82.0-88.0]$ & $<0.01$ \\
\hline $\operatorname{Men}(n=3475)$ & $1775(51.1)$ & $1160(58.0)$ & $290(51.1)$ & $325(35.8)$ & $<0.01$ \\
\hline \multicolumn{6}{|l|}{ Duration of HF $(n=3480)$} \\
\hline$<1$ year & $411(11.8)$ & $222(11.1)$ & $78(13.7)$ & $111(12.2)$ & \multirow[t]{3}{*}{0.17} \\
\hline $1-2$ years & 737 (21.2) & $407(20.3)$ & $126(22.2)$ & $204(22.5)$ & \\
\hline$\geq 2$ years & $2332(67.0)$ & $1376(68.6)$ & $364(64.1)$ & $592(65.3)$ & \\
\hline BMI, $\mathrm{kg} / \mathrm{m}^{2}(n=3139)$ & $25.0[23.0-29.0]$ & $25.0[23.0-28.0]$ & $26.0[23.0-29.0]$ & $26.0[24.0-30.0]$ & $<0.01$ \\
\hline \multicolumn{6}{|l|}{ NYHA $(n=3440)$} \\
\hline I & $335(9.7)$ & $189(9.5)$ & $44(7.8)$ & $102(11.4)$ & \multirow[t]{4}{*}{0.20} \\
\hline II & $1791(52.1)$ & $1036(52.3)$ & $310(55.1)$ & 445 (49.7) & \\
\hline III & $1217(35.4)$ & $705(35.6)$ & $195(34.6)$ & $317(35.4)$ & \\
\hline IV & $97(2.8)$ & $52(2.6)$ & $14(2.5)$ & $31(3.5)$ & \\
\hline LVEF, \% $(n=2326)$ & $40.0[30.0-50.0]$ & $30.0[25.0-35.0]$ & $45.0[40.0-47.0]$ & $58.0[52.0-60.0]$ & $<0.01$ \\
\hline \multicolumn{6}{|l|}{ Cause of HF $(n=3369)$} \\
\hline Ischemic cause of HF & $1518(45.1)$ & $1061(54.5)$ & $247(45.6)$ & $210(23.8)$ & \multirow[t]{2}{*}{$<0.01$} \\
\hline Non-ischemic cause of HF & $1851(54.9)$ & $885(45.5)$ & $295(54.4)$ & $671(76.2)$ & \\
\hline Systolic BP, mmHg $(n=3455)$ & $125.0[111.0-140.0]$ & $122.0[110.0-139.0]$ & $125.5[114.3-140.0]$ & $130.0[118.0-145.0]$ & $<0.01$ \\
\hline Diastolic BP, mmHg $(n=3461)$ & $70.0[60.0-76.0]$ & $70.0[60.0-75.0]$ & $70.0[60.0-76.0]$ & $70.0[60.0-79.0]$ & 0.01 \\
\hline Heart rate, bpm $(n=3446)$ & $70.0[63.0-80.0]$ & $70.0[63.0-80.0]$ & $71.0[62.0-82.0]$ & $70.0[63.0-80.0]$ & 0.42 \\
\hline Atrial fibrillation $(n=3445)$ & $1371(39.8)$ & $666(33.7)$ & $268(47.3)$ & $437(48.4)$ & $<0.01$ \\
\hline $\operatorname{LBBB}(n=3490)$ & $594(17.0)$ & $432(21.5)$ & 79 (13.9) & $83(9.1)$ & $<0.01$ \\
\hline $\mathrm{QRS} \geq 130 \mathrm{~ms}(n=2830)$ & $1131(40.0)$ & $786(48.5)$ & $167(35.8)$ & $178(24.0)$ & $<0.01$ \\
\hline $\operatorname{eGFR}(n=2459)$ & $44.2[32.4-60.6]$ & $45.2[33.1-61.2]$ & $43.4[31.6-58.5]$ & $42.0[31.2-59.6]$ & 0.03 \\
\hline \multicolumn{6}{|l|}{ eGFR $(n=2459)$} \\
\hline$<30$ & $498(20.3)$ & $283(18.9)$ & 87 (21.6) & $128(22.8)$ & \multirow[t]{3}{*}{0.18} \\
\hline $30-59$ & $1324(53.8)$ & $806(53.9)$ & $222(55.2)$ & $296(52.7)$ & \\
\hline$\geq 60$ & $637(25.9)$ & $406(27.2)$ & $93(23.1)$ & $138(24.6)$ & \\
\hline \multicolumn{6}{|l|}{ Comorbidity $(n=3158)$} \\
\hline Hypertension & $1485(47.0)$ & $763(42.2)$ & $237(46.1)$ & 485 (57.9) & $<0.01$ \\
\hline Diabetes mellitus & $862(27.3)$ & $495(27.4)$ & $139(27.0)$ & $228(27.2)$ & 0.98 \\
\hline COPD & $614(19.4)$ & $326(18.1)$ & $114(22.2)$ & $174(20.8)$ & 0.06 \\
\hline OSAS & $93(2.9)$ & $51(2.8)$ & $8(3.5)$ & $24(2.9)$ & 0.72 \\
\hline Thyroid disease & $259(8.2)$ & $153(8.5)$ & $31(6.0)$ & $75(8.9)$ & 0.13 \\
\hline No relevant comorbidity & $148(5.5)$ & $107(6.8)$ & $17(3.8)$ & $24(3.7)$ & $<0.01$ \\
\hline
\end{tabular}

$H F$ heart failure, $L V E F$ left ventricular ejection fraction, $E S C$ European Society of Cardiology, $H F r E F$ heart failure with reduced ejection fraction, $H F m r E F$ heart failure with mid-range ejection fraction, $H F p E F$ heart failure with preserved ejection fraction, $B M I$ body mass index, $N Y H A$ New York Heart Association classification, $B P$ blood pressure, $L B B B$ left-bundle branch block, $e G F R$ estimated glomerular filtration rate, $C O P D$ chronic obstructive pulmonary disease, OSAS obstructive sleep apnea syndrome 
[IQR] LVEF was 40\% [30.0-50.0], one quarter had diabetes mellitus and the majority $(74 \%)$ had an eGFR $<60 \mathrm{ml} /$ $\mathrm{min} / \mathrm{m}^{2}$ (Table 1). Several baseline characteristics differed significantly between LVEF groups, also when subdividing men and women (Suppl. Table 2). HFpEF patients $(n=911)$ were older and more often women, had a higher body mass index, more often had a non-ischemic cause of HF, hypertension, and atrial fibrillation in comparison to HFrEF patients $(n=2009)$. Octogenarians with HFrEF more often had a QRS-width $\geq 130 \mathrm{~ms}$ and left bundle branch block (LBBB) on their ECG, when compared to those with HFpEF, in those with sinus rhythm or atrial fibrillation, and not in HF patients with paced or ectopic rhythm (Table 1 and Suppl. Table 3).

Characteristics of HFmrEF patients aged $\geq 80$ years $(n=570)$ did not differ much from those with HFrEF except for a higher prevalence of atrial fibrillation and some other relevant comorbidities and fewer LBBB on ECG (Table 1). COPD was more prevalent in HFmrEF compared to HFrEF patients $(22.2 \%$ and $18.1 \%$, respectively, $p=0.04)$. HFpEF patients had more often hypertension when compared to both HFrEF and HFmrEF patients (Fig. 1).

\section{Guideline-recommended medical therapy in HFrEF}

Following the ESC guidelines 2016, a large proportion of HFrEF patients aged $\geq 80$ years received a beta blocker or a RAS inhibitor [angiotensin-converting enzyme (ACE) inhibitor or angiotensin receptor blocker (ARB)], i.e., $78.3 \%$ and $72.8 \%$, respectively. An MRA was prescribed in $52.0 \%$ of patients and diuretics in $90.4 \%$. Women received more often a beta blocker and a thiazide diuretic, than men (Table 2).

The combination of all three HF medication (beta blocker, RAS inhibitor and MRA), were prescribed to $29.9 \%$ of HFrEF patients aged $\geq 80$ years patients, two out of three HF medication in $46.5 \%$, one out of three in $20.3 \%$, and none of these medications were prescribed in $3.3 \%$ of octogenarians with HFrEF. In total, 55 patients $(2.7 \%)$ received ivabradine, which represents $77 \%$ of those where ivabradine was indicated.

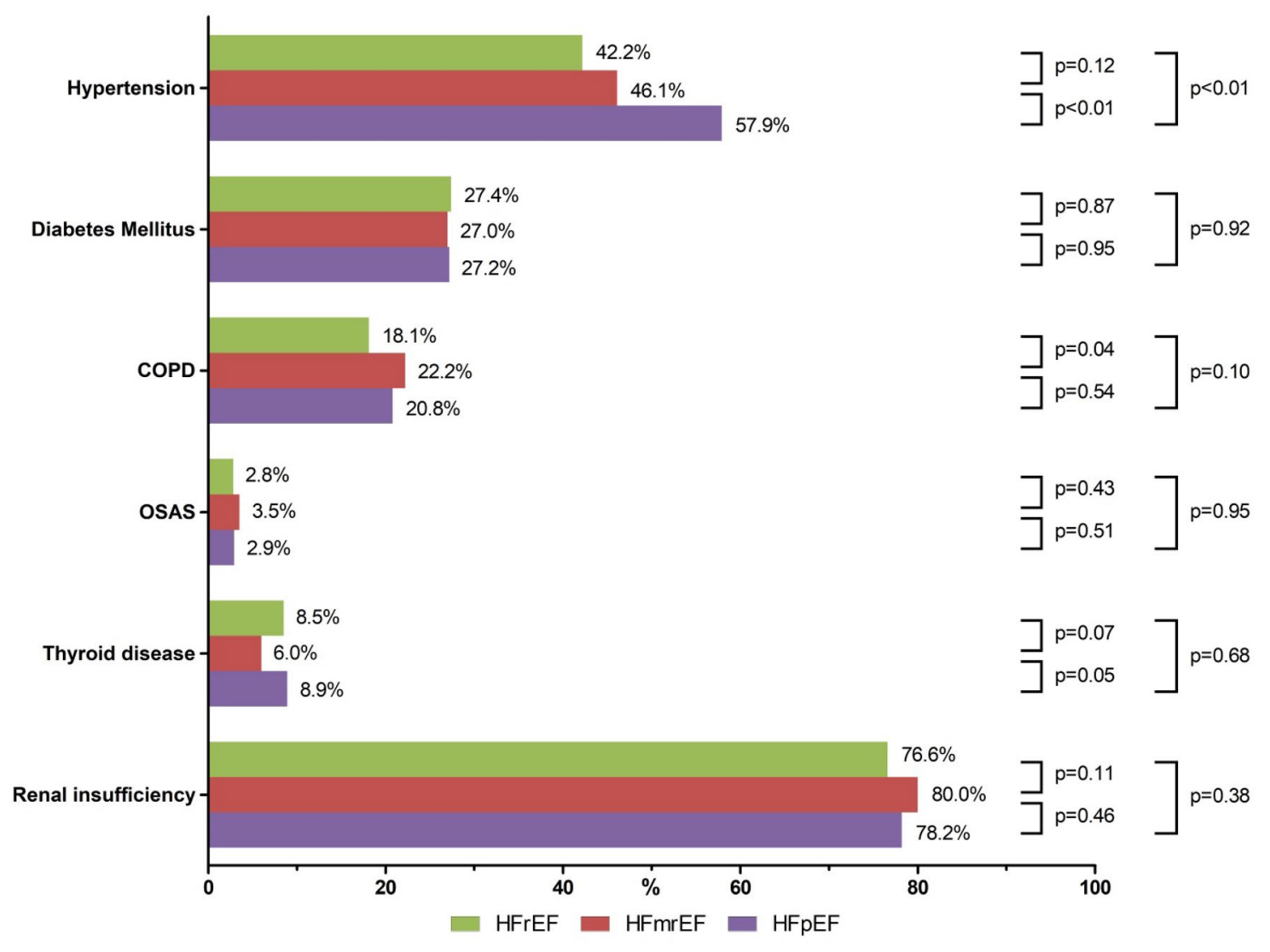

Fig. 1 Comorbidities in octogenarians with heart failure: HFrEF vs. HFmrEF vs. HFpEF (ESC Guidelines 2016). HFrEF heart failure with reduced ejection fraction, $H F m r E F$ heart failure with mid-range ejection fraction, $H F p E F$ heart failure with preserved ejection frac- tion, ESC European Society of Cardiology, COPD chronic obstructive pulmonary disease, OSAS obstructive sleep apnea syndrome. Renal insufficiency: defined as eGFR $<60 \mathrm{~mL} / \mathrm{min}$ or a history of renal failure 
Table 2 Percentage of HF therapy use in HFrEF, HFmrEF and HFpEF patients aged $\geq 80$ years, for men and women

\begin{tabular}{|c|c|c|c|c|c|c|c|c|}
\hline & & \multicolumn{5}{|c|}{ Guideline-recommended pharmacotherapy } & \multirow[t]{2}{*}{ Loop diuretics } & \multirow[t]{2}{*}{ Thiazide diuretics } \\
\hline & & Beta blocker & RAS inhibitor & MRA & Ivabradine & Diuretics & & \\
\hline \multirow[t]{3}{*}{ HFrEF } & Men & 879 (76.4) & $856(74.4)$ & $592(51.4)$ & $32(2.8)$ & 1035 (89.9) & $1,016(88.3)$ & $20(1.7)$ \\
\hline & Women & $672(81.2)$ & 585 (70.7) & 438 (52.9) & $23(2.7)$ & $754(91.1)$ & $731(88.3)$ & $31(3.7)$ \\
\hline & $p$ value & 0.01 & 0.07 & 0.52 & 0.98 & 0.40 & 0.99 & 0.01 \\
\hline \multirow[t]{3}{*}{ HFmrEF } & Men & $201(70.3)$ & $189(66.1)$ & $131(45.8)$ & $4(1.4)$ & $252(88.1)$ & $244(85.3)$ & $10(3.5)$ \\
\hline & Women & 206 (74.6) & $191(69.2)$ & $146(52.9)$ & $6(2.2)$ & 255 (92.4) & 251 (90.9) & $4(1.4)$ \\
\hline & $p$ value & 0.25 & 0.43 & 0.09 & 0.48 & 0.09 & 0.04 & 0.12 \\
\hline \multirow[t]{3}{*}{ HFpEF } & Men & $226(70.4)$ & 195 (60.7) & 144 (44.9) & $4(1.2)$ & 293 (91.3) & 277 (86.3) & $16(5.0)$ \\
\hline & Women & 437 (76.5) & $358(62.7)$ & $253(44.3)$ & $1(0.2)$ & $520(91.1)$ & $506(88.6)$ & $17(3.0)$ \\
\hline & $p$ value & 0.04 & 0.57 & 0.87 & 0.06 & 0.92 & 0.31 & 0.13 \\
\hline
\end{tabular}

$H F$ heart failure, $H F r E F$ heart failure with reduced ejection fraction, $H F m r E F$ heart failure with mid-range ejection fraction, $H F p E F$ heart failure with preserved ejection fraction, $R A S$ renin-angiotensin system, $R A S$ inhibitor angiotensin-converting enzyme (ACE) inhibitor or angiotensin receptor blocker (ARB), MRA mineralocorticoid receptor antagonists

MRA was less used in patients with more than 2 years of follow-up than in those with $<1$ year of HF follow-up, $49.0 \%$ and $61.5 \%$ respectively, $p<0.01$ (Suppl. Table $4 a$ ).

The percentages of target dose of HF medication prescribed in the 2009 HFrEF patients (LVEF $<40 \%)$ aged $\geq 80$ years are shown in Fig. 2. At least, $50 \%$ of target doses of beta blockers, RAS inhibitor and MRA were prescribed in $43.8 \%, 62.2 \%$ and $53.5 \%$ of HFrEF patients, respectively (Fig. 2). A $\geq 50 \%$ of target dose of all three of the HF medications groups was achieved in $9.5 \%$ of the patients $; \geq 50 \%$ of the target dose for two out of three HF medications in $35.9 \% ; \geq 50 \%$ of the target dose for one out of three HF medications in $39.2 \%$; and $\geq 50 \%$ of the target dose for none of these HF medications in $15.5 \%$.

The reasons of non-adherence or not prescribing recommended HF medication (ESC Guidelines 2016) were reported by the centers and are depicted in Table 3. Contraindications or intolerance for beta blockers was present in $3.5 \%$ of the patients, for RAS inhibitors, MRAs and ivabradine in, respectively, $7.2 \%, 6.1 \%$ and $2.2 \%$. There were no substantial differences between men and women (Suppl. Table 5). In a substantial number of patients, the

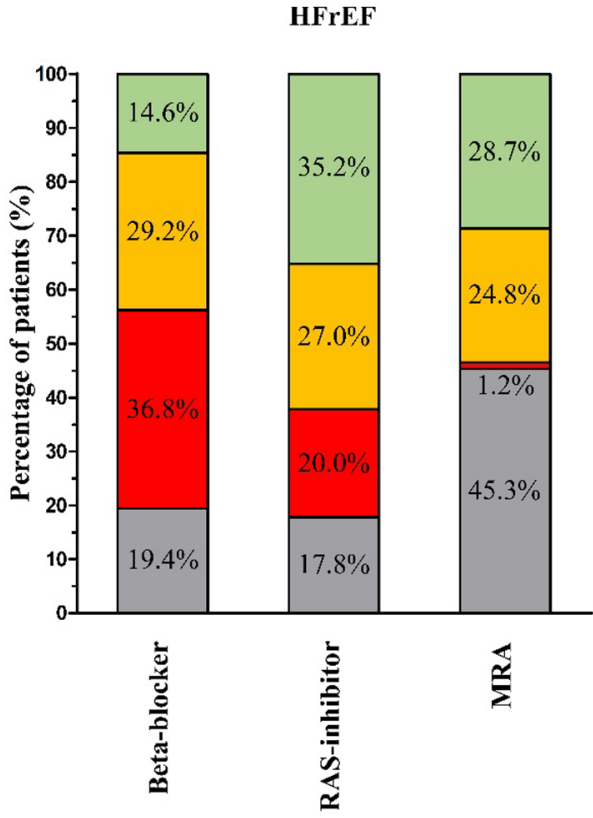

Fig. 2 Percentages of target dose of HF medication prescribed in octogenarians with $\mathrm{HFrEF}(\mathrm{LVEF}<40 \%)$. $H F$ heart failure, $\mathrm{HFrEF}$ heart failure with reduced ejection fraction, $R A S$ renin-angiotensin
HFmrEF

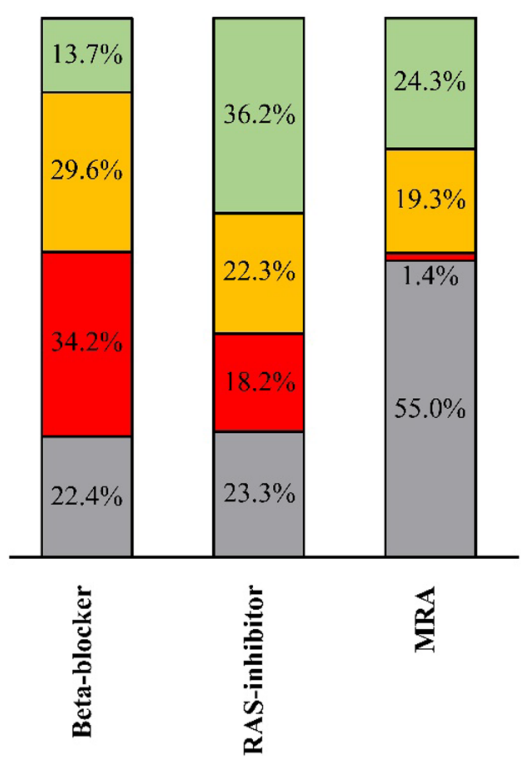

system, RAS inhibitor angiotensin-converting enzyme (ACE) inhibitor or angiotensin receptor blocker (ARB), MRA mineralocorticoid receptor antagonists 
Table 3 Reasons for not prescribing HF medication in HFrEF patients aged $\geq 80$ years

\begin{tabular}{lll}
\hline & $\begin{array}{l}\text { Contraindicated or } \\
\text { intolerance }\end{array}$ & No reason specified \\
\hline Beta blocker & $69(3.5)$ & $287(14.4)$ \\
RAS inhibitors & $145(7.2)$ & $396(19.9)$ \\
MRA & $121(6.1)$ & $834(41.9)$ \\
Ivabradine $^{\mathrm{a}}$ & $43(2.2)$ & $1891(95.1)$ \\
\hline
\end{tabular}

$H F$ heart failure, $H F r E F$ heart failure with reduced ejection fraction, $R A S$ renin-angiotensin system, $R A S$ inhibitor angiotensin-converting enzyme (ACE) inhibitor or angiotensin receptor blocker (ARB), $M R A$ mineralocorticoid receptor antagonists

${ }^{a}$ If indicated $(n=19) 25.7 \%$ of patients did not receive ivabradine with no specified reason

reasons for not receiving recommended HF-medication were not specified.

The results of multivariable analysis on guidelinedirected pharmacotherapy in octogenarians with HFrEF are presented in Table 4. Lower prescription rates of recommended RAS inhibitors were associated with higher age, NYHA class and heart rate, wider QRS, and also HFmrEF (versus HFrEF). Higher prescription rates of RAS inhibitors and diuretics were related to hypertension. Lower prescription of RAS inhibitors but higher use of beta blocker was associated with the presence of renal failure. MRA use was not associated with these comorbidities. Prescription rates of recommended HF medication were not independently associated with ischemic etiology of HF, diabetes mellitus 2 and COPD.

Digoxin was prescribed in one fifth of elderly HFrEF patients $(21.4 \%)$, amiodarone in $7.7 \%$ and statins in $69.8 \%$. Polypharmacy including beta blocker, RAS inhibitor, MRA, ivabradine, diuretics, statin, digoxin and amiodarone, median 4 of these drugs, was only slightly related to prescription of recommended beta blocker, RAS inhibitor and MRA (Suppl. Table 6).

\section{Medical treatment of HFmrEF patients}

In the 570 patients with $\mathrm{HFmrEF}$ aged $\geq 80$ years, beta blockers, RAS inhibitor and MRA were prescribed in $72.5 \%$, $67.6 \%$ and $49.1 \%$ of elderly HFmrEF patients, respectively. These proportions did not differ much from those in HFrEF patients (Table 2). Also, the percentages of the combined beta blocker, RAS inhibitor and/or MRA use in HFmrEF patients, aged $\geq 80$ years, were only slightly lower than in HFrEF octogenarians, for men and women (Fig. 3). Statins, digoxin and amiodarone were prescribed in $66.3 \%, 22.0 \%$ and $5.4 \%$ of HFmrEF patients, respectively.

\section{Medical treatment of HFpEF patients}

In the $911 \mathrm{HFpEF}$ patients aged $\geq 80$ years, diuretics were used by most frequently (91.2\%), followed by beta blockers (74.3\%), RAS inhibitors (62.0\%) and MRAs (44.6\%). Proportions of beta blockers, RAS inhibitors, MRA and diuretics did not differ much from those in HFrEF patients.
Table 4 Multivariable predictors of the use of $\mathrm{HF}$ medication in HFrEF patients aged $\geq 80$ years

\begin{tabular}{lllll}
\hline & Beta blocker & RAS inhibitor & MRA & Diuretics \\
& OR & OR & OR & OR \\
\hline Female gender & $1.31[1.02-1.68]$ & - & - & - \\
Age (per 10 years) & - & $0.63[0.48-0.83]$ & - & - \\
BMI (kg/m²) & - & - & - & $1.06[1.01-1.12]$ \\
Systolic BP (per 10 mmHg) & - & $1.09[1.02-1.16]$ & $0.80[0.75-0.85]$ & - \\
Diastolic BP (per 10 mmHg) & - & - & - & $0.77[0.63-0.93]$ \\
NYHA class (per class) & - & - & - & $2.07[1.49-2.88]$ \\
Heart rate (per 10 bpm) & - & - & - & - \\
QRS duration (per 10 ms) & - & $0.97[0.94-1.00]$ & - & - \\
eGFR (per 10 ml/min) & - & - & - & $0.85[0.76-0.94]$ \\
Ischemic etiology & - & - & - & - \\
Hypertension & - & - & - & $1.42[1.04-1.94]$ \\
Diabetes mellitus type 2 & - & - & - & - \\
COPD & - & - & - & - \\
Renal failure & - & $0.73[0.55-0.98]$ & - & $1.93[1.18-3.16]$ \\
\hline
\end{tabular}

$H F$ heart failure, $H F r E F$ heart failure with reduced ejection fraction, $R A S$ renin-angiotensin system, $R A S$ inhibitor angiotensin-converting enzyme (ACE) inhibitor or angiotensin receptor blocker (ARB), MRA mineralocorticoid receptor antagonists, $O R$ odds ratio, $B M I$ body mass index, $B P$ blood pressure, $N Y H A$ New York Heart Association classification, $e G F R$ estimated glomerular filtration rate, COPD chronic obstructive pulmonary disease, - variable not included in the model 


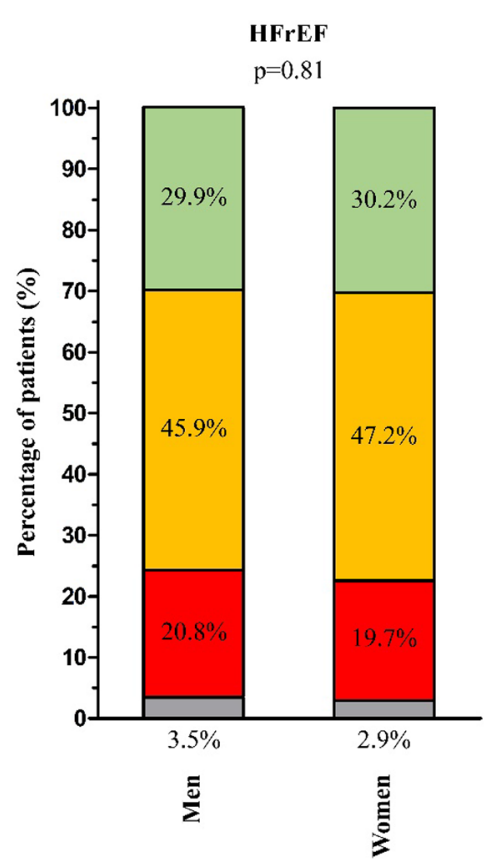

Fig. 3 Percentage of beta blocker, RAS inhibitor and/or MRA use in HFrEF and HFmrEF patients aged $\geq 80$ years for men and women. $H F r E F$ heart failure with reduced ejection fraction, $H F m r E F$ heart failure with mid-range ejection fraction, $R A S$ renin-angiotensin sys-

Digoxin was prescribed in one fifth of elderly HFpEF patients $(20.4 \%)$; and ivabradine and amiodarone in very few patients, $0.5 \%$ and $9.6 \%$, respectively.

The subgroup of HF with supernormal LVEF (LVEF $>65 \%$; HFsnEF) in our CHECK-HF octogenarians contained only 58 patients (1.7\%), see Suppl. Table 7. This new entity compromised too small patients group to make inferences and was not further included in the analyses.

\section{Discussion}

From our Dutch outpatient registry of chronic HF patients, we demonstrated that most octogenarians received recommended HF medication, although at lower percentages of target doses than previously reported in the entire group, except for MRAs [20]. Also, all three of the HF medications (beta blocker, RAS inhibitor and MRA) were prescribed in only about one quarter of octogenarians with HFrEF. Notably, women received more often beta blockers and thiazides than men.

The guidelines do not recommend specific HF therapies in patients with HFmrEF and HF medication did not differ significantly from HFrEF patients.

In the HFpEF group aged $\geq 80$ years, prescription rates of diuretics were higher than $90 \%$. A substantial proportion received also a beta blocker, RAS inhibitor or MRA, likely

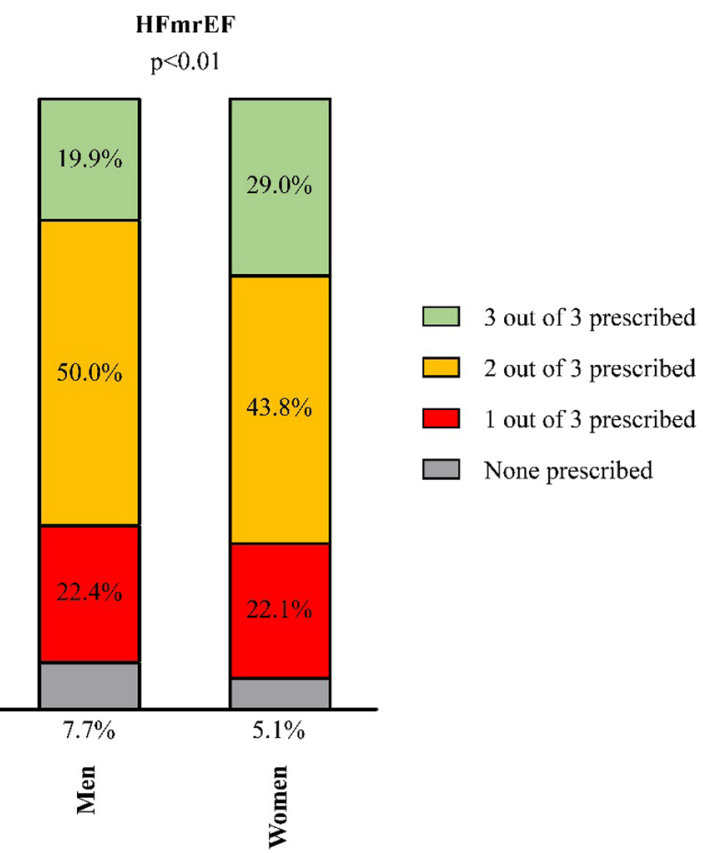

tem, RAS inhibitor angiotensin-converting enzyme (ACE) inhibitor or angiotensin receptor blocker (ARB), $M R A$ mineralocorticoid receptor antagonists, ESC European Society of Cardiology

to be related to the treatment of prevalent comorbidities. Due to clinical referral to out-hospital heart failure clinics, irrespective of patients' age, CHECK-HF contains a relatively high percentage of patients with HFrEF (overall 52\% and in octogenarians: 58\%) in comparison to the prevalence of HFpEF in Western populations. Although, the National Audit for England and Wales also reported that substantially more HFrEF than HFpEF patients $(66.8 \%$ and $33.2 \%$, respectively) were included in their registry (2016-2017), e.g., after a hospital admission for heart failure [21].

\section{Pharmacological therapy}

Elderly patients constitute a large part of the HF population in Western countries, 1-4 but only few studies addressed HF pharmacologic management of patients aged $\geq 80$ years [11-17]. In the Euro Heart Failure Survey (EHFS) II, both mortality rates of octogenarians during hospital stay and during follow-up of 12 months were significantly higher than in younger patients [12]. Notably, from the consecutive EHFS programs, a gradual improvement, though still suboptimal, of medical therapies in octogenarians hospitalized for HF was reported. The presence of comorbidities predicted mortality and the use of ACE inhibitors and beta blockers were associated with better outcome 11, 12]. The French OCTOCARDIO study found that even in the absence of comorbidity, in elderly patients with HF, ACE inhibitors 
and beta blockers were prescribed to only $40 \%$ and $48 \%$ of patients, respectively, probably because of their advanced age alone [13]. Data from a French national observational retrospective cohort of 1825 patients aged $>80$ years who were for the first time hospitalized for HF demonstrated that only $5 \%$ of them received an optimal treatment at discharge (combination of RAS inhibitor, beta blocker and MRA) [14]. During their follow-up period of 2 years, only beta-blocker prescription levels $(p=0.02)$ increased. In the CHECK-HF registry in chronic $\mathrm{HF}$, about one third of patients were aged $\geq 80$ years, thus resembling contemporary real-world practice in civilized countries. We found higher prescription rates of recommended HF medication than in these previous registries, which may be related to the delivery of specialist outpatient HF care in the vast majority of patients. However, in a substantial part of the HFrEF group, the actual dosages were lower than in younger patients [20].

Many factors may play a role in suboptimal therapy in the very old HF patients. In CHECK-HF, lower rates of guideline-directed pharmacotherapy in octogenarians with HFrEF were associated with NYHA class, LVEF and comorbidities. Lower prescription rates and tolerable dosages of recommended HF medication may be attributed to several limiting factors, e.g., low blood pressure and renal failure [27]. Also, recent data from the CHAMP-HF registry of in total 3518 patients from 150 primary care and cardiology practices showed that lower medication utilization or dose was associated with older age, lower blood pressure, more severe functional class, renal insufficiency, and recent HF hospitalization [18]. Remarkably, a recent post hoc analysis of the BIOSTAT-CHF study suggested that women with HFrEF might need lower doses of RAS inhibitors and beta blockers than men, also adjusted for age [22].

The Swedish Heart Failure Registry reported that $80 \%$ of HFrEF patients with age $>80$ years used RAS inhibitors, which was associated with reduced morbidity and mortality in this observational study [16]. Also, the use of beta blockers was associated with improved all-cause and CV survival [17]. So, suboptimal use of HF medication may lead to worse clinical outcomes. Also, only $40 \%$ patients of the total HFrEF cohort of that registry (11,215 patients, $27 \%$ women; mean age $75 \pm 11$ years) received a MRA [23]. Notably, the underuse of MRA was not related to hyperkalaemia, but among other factors, to impaired renal function, even in the range of a creatinine clearance $30-59.9 \mathrm{ml} / \mathrm{min}$, which is not a contraindication for MRA use. Adherence to guidelinedirected therapy of HFrEF, with prescription of at least $50 \%$ of the target dosage, is associated with better outcome [ 6 , 24-27]; although, this association has not been proven for very elderly HF patients. In the QUALIFY international registry, mainly younger patients were included and both mean age and age $\geq 74$ years did not influence adherence to (ESC 2012) guideline-directed medical therapy in HFrEF patients.
In addition, other age-related factors, particularly frailty, cognitive impairment and polypharmacy may contribute to suboptimal therapy of elderly HF patients [28]. In previous randomized clinical trials, patients aged $\geq 75$ years were underrepresented [7-10]. Consequently, there is no conclusive evidence that targeting at high dosages of medical therapy is equally beneficial in octogenarians compared with younger HFrEF patients and this may be another important reason for a lower uptake of HF medication in octogenarians. Awareness and assessment of comorbidities, and adequate management of these, may improve tailored HF care of the elderly patients [29]. In addition, reflection on optimal management and accepting lower age-adjusted target, tolerable dose of HF medication in elderly, may also be advocated. Accordingly, patient preferences and caregiver perceptions may influence therapeutic decisions in older HF patients [30].

In $\mathrm{HFpEF}$, there are unmet needs for evidence-based therapies in general and in elderly patients in particular, because of the steeply increasing prevalence with age. Interestingly, in the Swedish Heart Failure Registry, the use of RAS antagonists and beta blockers in HFpEF was associated with lower all-cause mortality [31, 32]. However, observational associations in HF have limited potential to make reliable therapeutic inferences, because (residual) confounding cannot be excluded [33].

\section{Limitations and strengths}

The CHECK-HF registry is a large-scale real-word registry of heart failure outpatient clinics in the Netherlands reflective of Western European countries. However, some limitations should be mentioned, such as the cross-sectional design and there were no outcome data collected. In addition, some missing data exist, which might influence results. However, imputation of missing data in multivariable analyses did not influence results. The etiology of heart failure was judged by the physician of the participating centers. Our registry included only patients seen in secondary, but not in primary care, which limits the generalizability of our findings to the primary care setting. Data on high age-related factors, e.g., frailty, cognitive impairment, dementia and disability were not collected, which may limit the understanding of the reason of not following the guidelines. Hardly any information was available for the use of sacubitril/valsartan, since it was approved in the Netherlands only in June 2016. Also, the use of oral nitrates (isosorbide-dinitrate or isosorbidemononitrate) combined with hydralazine is so low in the Netherlands that data was not collected. Strengths of the study are the reflection of the true practice of nationwide out-patient HF management and the high percentages of 
elderly patients with detailed information on medication prescription and dosage.

\section{Conclusion}

In this Dutch real-world registry of outpatient HF population, the majority of octogenarians received evidence-based HF medication, but at lower doses than recommended and only a minority received all three of the HF medication (beta blocker, RAS inhibitor (ACE inhibitor or ARB) and MRA). Analyses of clinical variables, including higher rates of comorbidities and reported contraindications and tolerances, did not fully explain the underuse of recommended HF therapies in octogenarians with HFrEF. Thus, future research should lead to strategies to improve management of elderly HF patients. Both in the HFmrEF group and the HFpEF group, in which evidence-based therapies are lacking, prescription rates of diuretics were also high and a substantial part of them received a beta blocker, RAS inhibitor and MRA.

Acknowledgements We greatly acknowledge the participation of heart failure nurses and cardiologists of all participating sites for including patients and entering patient data.

Funding Servier, the Netherlands, funded the inclusion of data and software program. The steering committee (HBRLR, GL, AH, JB) received no funding for this project. This analysis was initiated by the authors and was designed, conducted, interpreted, and reported independently of the sponsor.

\section{Compliance with ethical standards}

Conflict of interest The authors declare no competing financial interest.

\section{References}

1. Metra M, Teerlink JR (2017) Heart failure. Lancet 390(10106):1981-1995

2. van Deursen VM, Urso R, Laroche C, Damman K, Dahlström U, Tavazzi L et al (2014) Co-morbidities in patients with heart failure: an analysis of the European Heart Failure Pilot Survey. Eur J Heart Fail 16(1):103-111

3. Chioncel O, Lainscak M, Seferovic PM, Anker SD, Crespo-Leiro MG, Harjola VP et al (2017) Epidemiology and one-year outcomes in patients with chronic heart failure and preserved, midrange and reduced ejection fraction: an analysis of the ESC Heart Failure Long-Term Registry. Eur J Heart Fail 19(12):1574-1585

4. McMurray JJ, Adamopoulos S, Anker SD, Auricchio A, Böhm M, Dickstein K et al (2012) ESC Guidelines for the diagnosis and treatment of acute and chronic heart failure 2012: the task force for the diagnosis and treatment of acute and chronic heart failure 2012 of the European Society of Cardiology. Developed in collaboration with the Heart Failure Association (HFA) of the ESC. Eur Heart J 33(14):1787-1847
5. Ponikowski P, Voors AA, Anker SD, Bueno H, Cleland JG, Coats AJ et al (2016) 2016 ESC Guidelines for the diagnosis and treatment of acute and chronic heart failure: the task force for the diagnosis and treatment of acute and chronic heart failure of the European Society of Cardiology (ESC). Developed with the special contribution of the Heart Failure Association (HFA) of the ESC. Eur J Heart Fail 18(8):891-975

6. Komajda M, Cowie MR, Tavazzi L, Ponikowski P, Anker SD, Filippatos GS (2017) Physicians' guideline adherence is associated with better prognosis in outpatients with heart failure with reduced ejection fraction: the QUALIFY international registry. Eur J Heart Fail 19(11):1414-1423

7. Thomas S, Rich MW (2007) Epidemiology, pathophysiology, and prognosis of heart failure in the elderly. Clin Geriatr Med 23(1): $1-10$

8. Flather MD, Shibata MC, Coats AJ, van Veldhuisen DJ, Parkhomenko A, Borbola J et al (2005) Randomized trial to determine the effect of nebivolol on mortality and cardiovascular hospital admission in elderly patients with heart failure (SENIORS). Eur Heart J 26(3):215-225

9. Maggioni AP, Dahlstrom U, Filippatos G, Chioncel O, Crespo Leiro M, Drozdz J et al (2013) EURObservational Research Programme: regional differences and 1-year follow-up results of the Heart Failure Pilot Survey (ESC-HF Pilot). Eur J Heart Fail 15(7):808-817

10. Van Spall HG, Toren A, Kiss A, Fowler RA (2007) Eligibility criteria of randomized controlled trials published in high-impact general medical journals: a systematic sampling review. JAMA 297(11):1233-1240

11. Komajda M, Hanon O, Hochadel M, Follath F, Swedberg K, Gitt A et al (2007) Management of octogenarians hospitalized for heart failure in Euro Heart Failure Survey I. Eur Heart J 28(11):1310-1318

12. Komajda M, Hanon O, Hochadel M, Lopez-Sendon JL, Follath F, Ponikowski P et al (2009) Contemporary management of octogenarians hospitalized for heart failure in Europe: Euro Heart Failure Survey II. Eur Heart J 30(4):478-486

13. Moubarak G, Ernande L, Godin M, Cazeau S, Vicaut E, Hanon $O$ et al (2013) Impact of comorbidity on medication use in elderly patients with cardiovascular diseases: the OCTOCARDIO study. Eur J Prev Cardiol 20(4):524-530

14. Vorilhon C, Chenaf C, Mulliez A, Pereira B, Clerfond G, Authier $\mathrm{N}$ et al (2015) Heart failure prognosis and management in over-80-year-old patients: data from a French national observational retrospective cohort. Eur J Clin Pharmacol 71(2):251-260

15. Mizuno M, Kajimoto K, Sato N, Yumino D, Minami Y, Murai K et al (2016) Clinical profile, management, and mortality in veryelderly patients hospitalized with acute decompensated heart failure: an analysis from the ATTEND registry. Eur J Intern Med 27(1):80-85

16. Savarese G, Dahlström U, Vasko P, Pitt B, Lund LH (2018) Association between renin-angiotensin system inhibitor use and mortality/morbidity in elderly patients with heart failure with reduced ejection fraction: a prospective propensity scorematched cohort study. Eur Heart J 39(48):4257-4265

17. Stolfo D, Uij1 A, Benson L, Schrage B, Fudim M, Asselbergs FW et al. (2019) Association between beta-blocker use and mortality/morbidity in older patients with heart failure with reduced ejection fraction. A propensity score-matched analysis from the Swedish Heart Failure Registry. Eur J Heart Fail Sep. https:// doi.org/10.1002/ejhf.1615

18. Greene SJ, Butler J, Albert NM, DeVore AD, Sharma PP, Duffy CI et al (2018) Medical therapy for heart failure with reduced ejection fraction: the CHAMP-HF Registry. J Am Coll Cardiol 72(4):351-366 
19. Brugts JJ, Linssen GCM, Hoes AW, Brunner-La Rocca HP, Investigators of CHECK-HF (2018) Real-world heart failure management in 10,910 patients with chronic heart failure in the Netherlands: design and rationale of the Chronic Heart failure ESC guideline-based Cardiology practice Quality project (CHECK-HF) registry. Neth Heart J 26(5):272-279

20. Brunner-La Rocca HP, Linssen GC, Smeele FJ, van Drimmelen AA, Schaafsma HJ, Westendorp PH et al (2019) Contemporary drug treatment of chronic heart failure with reduced ejection fraction. The CHECK-HF registry. J Am Coll Cardiol HF 7(1):13-21

21. National Cardiac Audit Programme (2018) National Heart Failure Audit 2016/17 Summary Report. https://www.nicor.org.uk/wpcontent/uploads/2018/11/Heart-Failure-Summary-Report-201617.pdf

22. Santema BT, Ouwerkerk W, Tromp J, Sama IE, Ravera A, RegitzZagrosek V et al (2019) Identifying optimal doses of heart failure medications in men compared with women: a prospective, observational, cohort study. Lancet 394(10205):1254-1263

23. Savarese G, Carrero JJ, Pitt B, Anker SD, Rosano GMC, Dahlström U et al (2018) Factors associated with underuse of mineralocorticoid receptor antagonists in heart failure with reduced ejection fraction: an analysis of 11215 patients from the Swedish Heart Failure Registry. Eur J Heart Fail 20(9):1326-1334

24. Forman DE, Cannon CP, Hernandez AF, Liang L, Yancy C, Fonarow GC (2009) Influence of age on the management of heart failure: findings from Get With the Guidelines-Heart Failure (GWTG-HF). Am Heart J 157(6):1010-1017

25. Pfisterer M, Buser P, Rickli H, Gutmann M, Erne P, Rickenbacher P et al (2009) BNP-guided vs symptom-guided heart failure therapy: the Trial of Intensified vs Standard Medical Therapy in Elderly Patients With Congestive Heart Failure (TIME-CHF) randomized trial. JAMA 301(4):383-392

26. Brunner-La Rocca HP, Eurlings L, Richards AM, Januzzi JL, Pfisterer ME, Dahlström U et al (2015) Which heart failure patients profit from natriuretic peptide guided therapy? A meta-analysis from individual patient data of randomized trials. Eur J Heart Fail 17(12):1252-1261

27. Ouwerkerk W, Voors AA, Anker SD, Cleland JG, Dickstein K, Filippatos G et al (2017) Determinants and clinical outcome of uptitration of ACE-inhibitors and beta-blockers in patients with heart failure: a prospective European study. Eur Heart $\mathbf{J}$ 38(24):1883-1890

28. Vidan MT, Blaya-Novakova V, Sanchez E, Ortiz J, Serra-Rexach JA, Bueno H (2016) Prevalence and prognostic impact of frailty and its components in non-dependent elderly patients with heart failure. Eur J Heart Fail 18(7):869-875

29. Turner G, Clegg A (2014) Best practice guidelines for the management of frailty: a British Geriatrics Society, Age UK and Royal College of General Practitioners report. Age Ageing 43(6):744-748

30. Brunner-La Rocca HP, Rickenbacher P, Muzzarelli S, Schindler R, Maeder MT, Jeker U et al (2012) End-of-life preferences of elderly patients with chronic heart failure. Eur Heart J 33(6):752-759

31. Lund LH, Benson L, Dahlström U, Edner M (2012) Association between use of renin-angiotensin system antagonists and mortality in patients with heart failure and preserved ejection fraction. JAMA 308(20):2108-2117

32. Lund LH, Benson L, Dahlström U, Edner M, Friberg L (2014) Association between use of $\beta$-blockers and outcomes in patients with heart failure and preserved ejection fraction. JAMA 312(19):2008-2018

33. Rush CJ, Campbell RT, Jhund PS, Petrie MC, McMurray JJV (2018) Association is not causation: treatment effects cannot be estimated from observational data in heart failure. Eur Heart $\mathbf{J}$ 39(37):3417-3438

\section{Affiliations}

\section{Gerard C. M. Linssen ${ }^{1}$. Jesse F. Veenis ${ }^{2}$. Alexandra Kleberger ${ }^{1} \cdot$ Marcel J. W. Grosfeld $^{3}$ - Eric P. Viergever ${ }^{4}$. Bas M. van Dalen ${ }^{5}$. Wendy de Valk-Bedijn ${ }^{6}$. Jorina Langerveld ${ }^{7} \cdot$ Hans-Peter Brunner-La Rocca ${ }^{8} \cdot$ Arno W. Hoes $^{9}$. Jasper J. Brugts ${ }^{2}$. for the CHECK-HF investigators}

Gerard C. M. Linssen

g.linssen@zgt.nl

1 Department of Cardiology, Hospital Group Twente (Almelo and Hengelo), Zilvermeeuw 1, 7609 PP Almelo, The Netherlands

2 Department of Cardiology, Erasmus MC, University Medical Center Rotterdam, Rotterdam, The Netherlands

3 Department of Cardiology, Bernhoven, Uden, The Netherlands

4 Department of Cardiology, Groene Hart Ziekenhuis, Gouda, The Netherlands
5 Department of Cardiology, Franciscus Gasthuis \& Vlietland, Rotterdam, The Netherlands

6 Department of Cardiology, Martini Ziekenhuis, Groningen, The Netherlands

7 Department of Cardiology, Ziekenhuis Rivierenland, Tiel, The Netherlands

8 Department of Cardiology, Maastricht University Medical Centre, Maastricht, The Netherlands

9 Julius Center for Health Sciences and Primary Care, University Medical Center Utrecht and Utrecht University, Utrecht, The Netherlands 